\title{
Biermann battery mechanism and its role in evolution of astrophysical magnetic fields
}

\author{
Mikhailov E $\mathrm{A}^{* 1}$ and Andreasyan $\mathrm{R} \mathrm{R}^{2}$ \\ $1_{\text {M.V.Lomonosov Moscow State University, Moscow, Russia }}$ \\ ${ }^{2}$ V.A.Ambartsumian Byurakan Astrophysical Observatory, Byurakan, Armenia
}

\begin{abstract}
Nowadays it is well-known that a wide range of astrophysical objects have large-scale magnetic fields. Their observations are usually carried using Faraday rotation measurements. One of the possible mechanisms of their generation (at least the seed ones) can be connected with the Biermann battery mechanism. It is connected with difference between masses of protons and electrons, which are interacting with the cosmic medium. They produce the circular currents which can be generate the magnetic field which is perpendicular to the rotation plane. Here we present the mechanism of the magnetic field generation by the Biermann mechanism in the disc objects which can be useful for galaxies, accretion discs and another objects. One of the important features is connected with the influence of the existing magnetic fields (which can be induced by another charged particles) while studying the movement of the particles.
\end{abstract}

Keywords: magnetic fields, Biermann battery, galaxies

\section{Introduction}

Now it is well-known that a lot of different astrophysical objects have regular magnetic field structures (Zeldovich et al, 1983). There is a wide range of research of magnetic fields of the Sun, another stars, planets, galaxies, accretion discs and another objects. They are well studied using both observational and theoretical methods.

From the observational point of view, first studies of the magnetic fields in space were connected with description of dark spots. As for the galactic magnetic fields, first assumptions have been made by studying the cosmic rays. After that, the estimates of the magnetic field were done using the study of the synchrotron emission spectra (Fermi, 1949). Nowadays most of the studies are done using measurements of the Faraday rotation of the polarization plane of the radio waves (Beck et al, 1996; Arshakian et al, 2009). For the Milky Way, such research is usually done basing on the data about pulsars (Andreasyan et al, 2020). As for another galaxies, the extragalactic sources are used. Sometimes they are taken for our Galaxy, too (Oppermann et al, 2012) Also the Faraday rotation is useful to study the magnetic fields of another cosmic objects.

As for the theory, the magnetic fields are usually described by the dynamo mechanism (Beck et al, 1996). It is based (Moffatt, 1978) on joint activity of the differential rotation (which is connected with the non-solid rotation of the galaxies) and alpha-effect (it describes the vorticity of the turbulent motions of the interstellar medium). If they are more intensive than the turbulent diffusivity, the magnetic field will grow according to the exponential law. However, it is necessary to have the initial field. It is very difficult to describe it using only dynamo, so we should take another processes.

One of the possible mechanisms is connected with so-called Biermann battery (Biermann, 1950). Principally it is based on outflows from the central objects to the outer parts (Andreasyan, 1996). They contain protons and electrons which interact with the rotating medium. The different masses of these particles make them interact in different way. So, the velocity of them is different and there

*ea.mikhajlov@physics.msu.ru, Corresponding author 
are circular currents. Such currents produce magnetic fields which can play a great role in galactic magnetism.

It is important to take into account, that every particle moves in the existing magnetic field. For example, this field can be connected with the magnetic fields of another particles. So, the growth of the magnetic field can be limited by the interaction between the particles and the existing magnetic field.

Here we present the model for the motion of the particles and estimate the typical values of the velocity and circular currents. Also we calculate the magnetic field induced by it.

\section{Basic equations and their solution}

We can consider the motion of the charged particle which outflows from the central body in the galaxy or another object of disc shape (fig. 1). The acceleration $\mathbf{w}$ of the particle will be the following:

$$
\mathbf{w}=-\frac{1}{\tau}(\mathbf{v}-\mathbf{V})+\mathbf{F}_{L}+\mathbf{F}_{g}
$$

where $\mathbf{v}$ is the velocity of the particle, $\mathbf{V}$ is the velocity of the medium, $\tau$ is the typical timescale of the interaction between the rotating medium and the particle, $\mathbf{F}_{g}$ is the gravitational force and $\mathbf{F}_{L}$ is the Lorentz force.

It is quite reasonable to assume, that the radial velocity of the particle has the uniform value $V$. Also, the magnetic field is directed vertically, and the Lorentz force will be:

$$
F_{L}=\frac{q}{m c} \dot{r} B
$$

So, the main component of the equation is associated with $\varphi$-component:

$$
w_{\varphi}=-\frac{1}{\tau} r(\dot{\varphi}-\Omega)-\frac{q}{m c} \dot{r} B
$$

As for the component of the acceleration we shall have:

$$
w_{\varphi}=r \ddot{\varphi}+2 \dot{r} \dot{\varphi}
$$

The equation for the angle evolution will be the following:

$$
r \ddot{\varphi}+2 \dot{r} \dot{\varphi}=-\frac{r}{\tau}(\dot{\varphi}-\Omega)-\frac{q}{m c} \dot{r} B .
$$

We can take into account that $\dot{r}=V$ and assume that the distance from the center changes quite slowly. So we can rewrite the equation as:

$$
\ddot{\varphi}=-\frac{2 V}{R} \dot{\varphi}-\frac{1}{\tau}(\dot{\varphi}-\Omega)-\frac{q}{R m c} V B .
$$

This equation can be rewritten as:

$$
\begin{gathered}
\ddot{\varphi}+\left(\frac{2 V}{R}+\frac{1}{\tau}\right) \dot{\varphi}=\frac{\Omega}{\tau}-\frac{q V B}{R m c} \\
\frac{d}{d t}\left(\dot{\varphi} \exp \left(\left(\frac{2 V}{R}+\frac{1}{\tau}\right) t\right)\right)=\left(\frac{\Omega}{\tau}-\frac{q V B}{R m c}\right) \exp \left(\left(\frac{2 V}{R}+\frac{1}{\tau}\right) t\right) .
\end{gathered}
$$




\section{Solutions of the equations}

Both parts of the equation can be integrated as:

$$
\dot{\varphi} \exp \left(\left(\frac{2 V}{R}+\frac{1}{\tau}\right) t\right)+C=\frac{\left(\frac{\Omega}{\tau}-\frac{q V B}{R m c}\right) \exp \left(\left(\frac{2 V}{R}+\frac{1}{\tau}\right) t\right)}{\frac{2 V}{R}+\frac{1}{\tau}} ;
$$

where $C$ is some constant connected with the initial conditions.

If in the beginning $\varphi=0$, we can take that:

$$
C=\frac{\left(\frac{\Omega}{\tau}-\frac{q V B}{R m c}\right)}{\frac{2 V}{R}+\frac{1}{\tau}}
$$

so the equation for the velocity will be:

$$
\dot{\varphi} \exp \left(\left(\frac{2 V}{R}+\frac{1}{\tau}\right) t\right)+\frac{\left(\frac{\Omega}{\tau}-\frac{q V B}{R m c}\right)}{\frac{2 V}{R}+\frac{1}{\tau}}=\frac{\left(\frac{\Omega}{\tau}-\frac{q V B}{R m c}\right) \exp \left(\left(\frac{2 V}{R}+\frac{1}{\tau}\right) t\right)}{\frac{2 V}{R}+\frac{1}{\tau}} ;
$$

For the derivative of the angle we shall have:

$$
\dot{\varphi}=\frac{\left(\frac{\Omega}{\tau}-\frac{q V B}{R m c}\right)}{\frac{2 V}{R}+\frac{1}{\tau}}\left\{1-\exp \left(-\left(\frac{2 V}{R}+\frac{1}{\tau}\right) t\right)\right\} .
$$

If we are speaking about the radial velocity, it will come to the value:

$$
\dot{\varphi}=R \frac{\Omega-\frac{q \tau V B}{R m c}}{1+\frac{2 V \tau}{R}}\left\{1-\exp \left(-\frac{t}{\tau}\left(1+\frac{2 V \tau}{R}\right)\right)\right\} .
$$

So the velocity will reach the limit value:

$$
v_{\varphi}=V_{0}\left\{1-\exp \left(-\frac{t}{T}\right)\right)
$$

where

$$
\begin{gathered}
V_{0}=R \frac{\Omega-\frac{q \tau V B}{R m c}}{1+\frac{2 V \tau}{R}} ; \\
T=\frac{\tau}{1+\frac{2 V \tau}{R}} .
\end{gathered}
$$

The time period $T$ is usually much smaller than another typical times of the problem, so the azimuthal velocity soon becomes close to $V_{0}$. Taking into account that $\frac{2 V \tau}{R} \ll 1$, for the velocity we shall have:

$$
v_{\varphi} \approx R \frac{\Omega-\frac{q \tau V B}{R m c}}{1-\frac{2 V \tau}{R}} \approx R \Omega-2 V \Omega \tau-\frac{q \tau V B}{m c} .
$$

The typical period of the particle is the following:

$$
T=\frac{2 \pi R}{v}=\frac{2 \pi}{\Omega-\frac{2 V \Omega \tau}{R}-\frac{q \tau V B}{R m c}}
$$

It is connected with the current:

$$
I=\frac{q}{T}=\frac{q}{2 \pi}\left(\Omega-\frac{2 V \Omega \tau}{R}-\frac{e \tau V B}{R m c}\right)
$$

We describe two different types of particles. For the protons we will have:

$$
I_{p}=\frac{e}{2 \pi}\left(\Omega-\frac{2 V \Omega \tau_{p}}{R}-\frac{e \tau_{p} V B}{R m_{p} c}\right)
$$




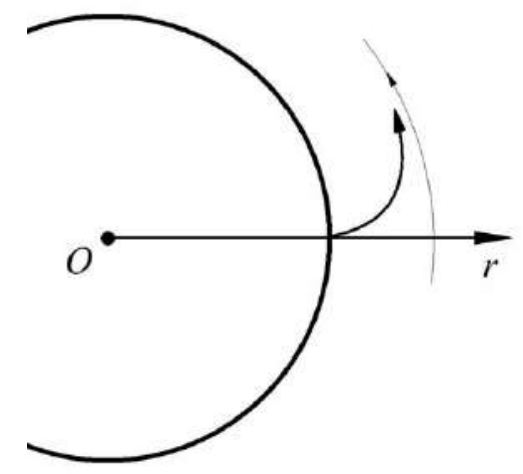

Figure 1. Motion of the particle.

where $\tau_{p}$ is the typical collision time for protons, $m_{p}$ is the proton mass. For electrons:

$$
I_{e}=-\frac{e}{2 \pi}\left(\Omega-\frac{2 V \Omega \tau_{e}}{R}+\frac{e \tau_{e} V B}{R m_{e} c}\right)
$$

where $\tau_{e}$ is the typical electron collision time, $m_{e}$ is the mass of the electron. Each pair corresponds to the full current:

$$
I=I_{p}+I_{e}=-\frac{e V \Omega\left(\tau_{p}-\tau_{e}\right)}{\pi R}-\frac{e^{2} V B}{\pi R c}\left(\frac{\tau_{p}}{m_{p}}+\frac{\tau_{e}}{m_{e}}\right) .
$$

If we take into account that $\tau$ is proportional to squared mass, the part which is connected with the electrons can be neglected, and the current will be the following:

$$
I=-\frac{e V \Omega \tau_{p}}{\pi R}-\frac{e^{2} V B \tau_{p}}{\pi R c m_{p}} .
$$

This current corresponds to the magnetic momentum:

$$
m=\frac{I S}{c}
$$

where $S=\pi R^{2}$, so

$$
m=-\frac{e V \Omega R}{c}-\frac{e^{2} V B \tau_{p} R}{c^{2} m_{p}} .
$$

The first part corresponds to the effect, which is connected with the linear mechanism (Andreasyan, 1996). The second is connected with the influence of the magnetic field. This field can be connected both with the external part and the self magnetic field induced by such particles.

\section{Conclusion}

We have studied the role of the Biermann battery mechanism in the evolution of the magnetic fields of objects of disc geometry. We have taken into account the influence of the existing magnetic field. Such mechanism can describe the saturation of the magnetic field growth for this case. The full magnetic field generated by the Biermann battery can be described by the self-consistent model, which takes into account the integral effect of the growth of the magnetic momentum.

\section{References}

Andreasyan R. R., 1996, Astrophysics, 39, 58

Andreasyan R. R., Mikhailov E. A., Andreasyan H. R., 2020, Astronomy Reports, 64, 189 
Arshakian T., Beck R., Krause M., Sokoloff D., 2009, Astronomy and Astrophysics, 494, 21

Beck R., Brandenburg A., Moss D., et al. 1996, Annual Review of Astronomy and Astrophysics, 1, 155

Biermann L., 1950, Zeitschrift Naturforschung Teil A, 5, 65

Fermi E., 1949, Physical Review, 75, 1169

Moffatt H., 1978, Magnetic field generation in electrically conducting fluids. University Press, Cambridge

Oppermann E., Junklewitz H., Robbers G. e. a., 2012, Astronomy and Astrophysics, 542, A93

Zeldovich Y., Ruzmaikin A. A., Sokoloff D. D., 1983, Magnetic Fields in Astrophysics. Gordon and Breach, New York 